Artículo científico

(Original paper)

\title{
CEBOS FEROMONALES PARA LA CAPTURA DE SPODOPTERA FRUGIPERDA (J. E SMITH) (LEPIDOPTERA: NOCTUIDAE) EN CULTIVOS DE MAÍZ ADYACENTES A CULTIVOS DE FRESAS
}

\author{
PHEROMONAL BAITS FOR THE CAPTURE OF SPODOPTERA FRUGIPERDA (J. E. SMITH) \\ (LEPIDOPTERA: NOCTUIDAE) IN MAIZE CROPS ADJACENT TO STRAWBERRY CROPS
}

\author{
Samuel CRUZ-eSteban ${ }^{1,2 *}$, Patricia HeRnándeZ-LedeSMA ${ }^{1}$, Edi A. MAlO ${ }^{3}$, Julio C. ROJAS ${ }^{3}$ \\ ${ }^{1}$ Instituto de Ecología, A.C. Red de Diversidad Biológica del Occidente Mexicano. Avenida Lázaro Cárdenas 253, \\ 61600 Pátzcuaro, Michoacán, México.<samuel.cruz@inecol.mx>; <patricia.hernandez@inecol.mx> \\ ${ }^{2}$ CONACyT. Avenida Insurgentes Sur 1582, 03940 Ciudad de México, México. <samuel.cruz@inecol.mx> \\ ${ }^{3}$ Departamento de Entomología Tropical, de El Colegio de la Frontera Sur, Carretera Antiguo Aeropuerto Km 2.5, \\ 30700 Tapachula, Chiapas, México.<emr@ecosur.mx>; <jrojas@ecosur.mx> \\ *Autor corresponsal: <samuel.cruz@inecol.mx>
}

Recibido: 14/11/2019; aceptado: 15/01/2020; publicado en línea: 10/02/2020

Editor responsable: Magdalena Cruz

Cruz-Esteban, S., Hernández-Ledesma, P., Malo, E. A., Rojas, J. C. (2020) Cebos feromonales para la captura de Spodoptera frugiperda (J. E. Smith) (Lepidoptera: Noctuidae) en cultivos de maíz adyacentes a cultivos de fresas. Acta Zoológica Mexicana (nueva serie), 36, 1-15. https://doi.org/10.21829/azm.2020.3612255

RESUMEN. El gusano cogollero, Spodoptera frugiperda (J. E. Smith) (Lepidoptera: Noctuidae), es una de las principales plagas del maíz y el sorgo, además de que este insecto empieza a ser una amenaza para el cultivo de fresa en Michoacán, México. Debido al daño que puede causar este insecto, es necesario contar con un sistema de monitoreo de sus poblaciones para detectar infestaciones tempranas e implementar su control a tiempo. Bajo este escenario, el uso de trampas cebadas con feromona sexual puede ser útil para atraer a los machos. En este estudio, se comparó la efectividad de una feromona desarrollada a partir de poblaciones mexicanas de $S$. frugiperda contra cuatro formulaciones comerciales, tres formuladas en el extranjero y una en México, en cultivos de maíz adyacentes a cultivos de fresas. También, se evaluó el efecto del color de la trampa en las capturas de $S$. frugiperda, usando uno de los cebos que presentó mayores capturas. Las trampas cebadas con cebo optimizado para poblaciones mexicanas (EcoTap) y con el cebo comercial formulado en el país (Phero-SF) fueron las que capturaron más machos de S. frugiperda y menos insectos no blancos, comparado con las trampas cebadas con los otros cebos. Uno de los hallazgos inesperados fue la captura de machos del gusano cortador, Agrotis ipsilon (Hufnagel) (Lepidoptera: Noctuidae), por las trampas cebadas con EcoTap o Phero-SF. El color de la trampa no fue una variable significativa para la captura de machos de S. frugiperda o A. ipsilon. Estos resultados pueden ser de importancia para el desarrollo de un sistema de monitoreo o trampeo masivo de $S$. frugiperda en las regiones mexicanas donde el maíz y sorgo son los principales cultivos, o en aquellos orgánicos de fresas que pueden estar amenazados por este insecto polífago.

Palabras clave: gusano cogollero; comunicación sexual; trampas de color; Agrotis ipsilon 
Cruz-Esteban, S., Hernández-Ledesma, P., Malo, E. A., Rojas, J. C. (2020) Pheromonal baits for the capture of Spodoptera frugiperda (J. E. Smith) (Lepidoptera: Noctuidae) in maize crops adjacent to strawberry crops. Acta Zoológica Mexicana (nueva serie), 36, 1-15. https://doi.org/10.21829/azm.2020.3612255

\begin{abstract}
The fall armyworm, Spodoptera frugiperda (J. E. Smith) (Lepidoptera: Noctuidae), is one of the main pests of maize and sorghum, in addition this insect begins to be a threat for strawberry crops in Michoacán, Mexico. Thus, it would be worth to have a monitoring system to detect early infestations of $S$. frugiperda and implement their control in time. Under this scenario, the use of traps baited with the sex pheromone may be useful to catch males. In this study, we compared the effectiveness of a pheromone lure developed from Mexican populations of $S$. frugiperda against four commercial lures, three formulated abroad and one in Mexico, in maize crops adjacent to strawberries ones. Additionally, we analyzed the effect of the trap color. It was found that the traps baited with lure optimized for Mexican populations (EcoTap) or with the commercial lure formulated in Mexico (Phero-SF) captured more $S$. frugiperda males and less non-target insects compared to traps baited with the other lures. One unexpected finding was the caught of black cutworm males, Agrotis ipsilon (Hufnagel) (Lepidoptera: Noctuidae), by the traps baited with EcoTap or Phero-SF lures. The trap colour did not have a significant effect on the capture of $S$. frugiperda or $A$. ipsilon males. The results obtained here may be used for monitoring or mass trapping S. frugiperda in the Mexican regions where maize and sorghum are the main crops or in organic strawberry crops that may be threatened by this polyphagous insect.
\end{abstract}

Key words: fall armyworm; communication sexual; colour traps; Agrotis ipsilon

\title{
INTRODUCCIÓN
}

El gusano cogollero, Spodoptera frugiperda (J. E. Smith) (Lepidoptera: Noctuidae), es un insecto altamente polífago que ataca a una amplia variedad de plantas de diferentes familias, pero muestra especial preferencia por las gramíneas (Casmuz et al., 2010; Montezano et al., 2019). Actualmente, este insecto es una de las principales limitantes en la productividad del maíz en todo el mundo (Oerke, 2006). En México, es una de las plagas insectiles más importante del cultivo de maíz (Malo et al., 2001; Blanco et al., 2014; Aguirre et al., 2016). Sin embargo, se ha observado recientemente el ataque de este insecto en algunos cultivos de fresas en el estado de Michoacán, México (Cruz-Esteban, datos no publicados), tal y como se ha reportado en Colombia (Solano et al., 2015). Este insecto tiene una alta capacidad migratoria, por lo que se encuentra distribuido en todo el continente americano (Andrews, 1988; Prowell et al., 2004; Clark et al., 2007; Hardke et al., 2011; Pashley, 1986), además de que se ha convertido en una plaga dañina e invasiva del maíz en algunos países de África (Goergen et al., 2016; Stokstad, 2017) y Asia (Ganiger et al., 2018; Shylesha et al., 2018; Swamy et al., 2018).

El maíz es un cultivo ampliamente utilizado en la dieta tradicional de los mexicanos. México es el área de origen y domesticación del maíz y, actualmente, es el quinto productor de este cereal a nivel mundial (Matsuoka et al., 2002). Por otro lado, México ocupa el tercer lugar mundial como productor de fresas (SENASICA, 2019) y Michoacán es uno de los estados en donde se concentra la mayor producción de estos frutos, lo que constituye un valor fundamental para la economía agrícola de este estado y del país. Aunque actualmente $S$. frugiperda no representa un daño económico al cultivo de la fresa, es de gran importancia monitorearlo, ya que se trata de un insecto altamente adaptable y resistente a los diferentes hospederos y cambios ambientales. Posiblemente, ésta sea la razón por la cual se ha encontrado alimentándose de los brotes de plantas de fresas.

El manejo de $S$. frugiperda se basa principalmente en la aplicación de insecticidas químicos (piretroides y organofosforados) o biológicos (Bacillus thuringiensis) (Tamez et al., 2001; Blanco et al., 
2014). En la actualidad, a pesar del impacto ambiental negativo, así como los efectos adversos para la salud humana y el medio ambiente, los insecticidas órgano-sintéticos constituyen el principal método de control de esta plaga. Sin embargo, su éxito es limitado, debido a que las larvas se ubican principalmente en los brotes de la planta huésped, quedando fuera del alcance de los insecticidas. Por lo que se requieren medios de control más eficaces y ecológicamente aceptables (Batista-Pereira et al., 2006).

El control etológico es una estrategia prometedora para el manejo de insectos plagas. En este caso, se basa en la comunicación química intraespecífica por feromona sexual que los insectos utilizan para encontrarse y reproducirse, y por la detección de señales químicas (kairomonas) para ubicar a las plantas y frutos hospederos. Al aprovechar la respuesta de los insectos a estos estímulos en sus propios ambientes naturales, se pueden diseñar cebos atractivos y cebos alimenticios tóxicos, así como trampas que pueden servir para el monitoreo, trampeo masivo, atracción-aniquilación o dispositivos para la interrupción del apareamiento de poblaciones de plagas insectiles. En varias especies de palomillas, la comunicación sexual está mediada por feromonas, las cuales pueden presentar variación a nivel geográfico entre las poblaciones de una determinada especie (Tòth et al., 1992; Gemeno et al., 2000; Groot et al., 2009). Esta variación incluye cambios, ya sean cualitativos o cuantitativos de los componentes de feromonas sexuales, o cambios en las respuestas de los machos (Phelan, 1992; Baker, 2002; Smadja \& Butlin, 2009).

En los extractos de las glándulas sexuales de las hembras de $S$. frugiperda de poblaciones de Florida (Estados Unidos), Guayana Francesa y Brasil, se han identificado varios acetatos, incluido el acetato de (Z)9-tetradecenilo (Z9-14:OAc) como componente principal, el acetato de (Z)-7-dodecenilo (Z7-12:OAc), el acetato de (Z)-11-hexadecenilo (Z11-16:OAc), el acetato de (Z)-9-dodecenilo (Z9-12:OAc) y el acetato de (E)-7-dodecenilo (E7-12:OAc) (Tumlinson et al., 1986; Descoins et al., 1988; Batista-Pereira et al., 2006). El último compuesto solo se ha encontrado en poblaciones brasileñas de $S$. frugiperda (Batista-Pereira $e t$ al., 2006). En poblaciones mexicanas, los compuestos emitidos por las hembras en el momento del llamado sexual contienen tres acetatos: Z9-14:OAc como componente mayoritario, Z11-16:OAc como componente secundario y Z7-12:OAc como trazas (Cruz-Esteban et al., 2018). Actualmente, se ha encontrado que para capturar machos de $S$. frugiperda en campos de maíz en Costa Rica, Canadá, Estados Unidos (Florida, Carolina del Norte, Puerto Rico), Perú, Argentina y México, solo se necesitan dos de estos acetatos, el Z914:OAc y Z7-12:OAc (Andrade et al., 2000; Fleischer et al., 2005; Unbehend et al., 2014; Cruz-Esteban et al., datos no publicados).

La variación geográfica en la comunicación sexual de $S$. frugiperda puede tener un impacto en el éxito o fracaso en el manejo de esta plaga agrícola mediante feromonas, tal como se ha demostrado en otras especies de palomillas (Cardé \& Minks, 1995; El-Sayed et al., 2009; Witzgall et al., 2010). Al utilizar feromonas comerciales que han sido optimizadas con poblaciones de $S$. frugiperda diferentes a las de nuestro país, ha resultado en capturas erráticas (Malo et al., 2001). Por lo que es más recomendable utilizar feromonas sexuales optimizadas a partir de poblaciones de las regiones donde se realiza el programa de monitoreo o de trampeo masivo, tal como se ha realizado con otros lepidópteros (Mafra-Neto \& Habid,1996; Kamarudin et al., 2010; Larraín et al., 2009).

El objetivo de este trabajo fue comparar la efectividad de una feromona sexual que ha sido optimizada a partir de poblaciones de $S$. frugiperda de varios estados de México en cultivos de maíz, contra cuatro feromonas sexuales comerciales, tres formuladas en otros países y otra formulada en nuestro país. Por otro lado, como el efecto del color en la atracción de las palomillas aún no es del todo claro, se evaluó este factor para optimizar un sistema de captura basado en trampa-atrayente sexual en el estado de Michoacán, pero que puede ser útil para todo el país contra esta plaga. 


\section{MATERIALES Y MÉTODOS}

Área de estudio. Las pruebas de campo se realizaron en Tiripetío $\left(19^{\circ} 31^{\prime} 55^{\prime \prime} \mathrm{N}, 101^{\circ} 22^{\prime} 10\right.$ ” O, a 2,020 $\mathrm{msnm}$ ), localizado en el extremo sur del municipio de Morelia, Michoacán, donde se desarrollan cultivos de frutos suaves, principalmente arándano (Vaccinium corymbosum, Ericaceae), frambuesa (Rubus idaeus, Rosaceae) fresa (Fragaria spp., Rosaceae), y zarzamora (Rubus ulmifolius, Rosaceae). Los predios comerciales con estos cultivos pertenecen a Koppert Development Institute Berries. Una de las estrategias de esta empresa, es dar un año de descanso a las tierras después de ciertos años de cultivo (dependiendo del cultivo): las áreas cultivadas por fresa después de un año de cultivo, las cultivadas por frambuesa después de dos años, las cultivadas por zarzamora después de 10 años y las cultivadas por arándano después de 14 años. Estas áreas de descanso siempre son cultivadas por maíz (híbrido de Ceres) o una mezcla de leguminosas fijadoras de nitrógeno. Las dos pruebas de campo (efecto de la feromona sexual y del color de trampa) se realizaron en los perímetros de las áreas cultivadas de maíz y de fresas.

Efecto de la feromona sexual en las capturas. En el primer experimento se evaluó el efecto de la feromona sexual en la captura de machos de S. frugiperda. Para este experimento, se evaluó la feromona optimizada por Cruz-Esteban et al. (datos no publicados), con un dispensador de caucho rojo que se le denominó "EcoTap" contra las feromonas comerciales: Scentry (Scentry Inc., Buckeye, Arizona), con un dispensador de caucho gris; Trécé (Trécé Inc., Salinas, California), con un dispensador de caucho rojo, obtenidos a través de Gempler's Inc., Belleville, WI, (EUA); ChemTica, una burbuja contenida en un sobre de polímero (Chemtica, Heredia, Costa Rica), y Phero-SF (Squid Biological and Pheromones S.A. de C.V., México), con un dispensador de caucho gris. Las trampas usadas en este experimento fueron elaboradas con garrafas de plástico color amarillo de $3.78 \mathrm{~L}$ de capacidad (Malo et al., 2018), a cada una de éstas se les cortó tres ventanas laterales (dos de $15 \times 11 \mathrm{~cm}$, y una de $10 \times 11 \mathrm{~cm}$ ), y se les agregó agua con jabón al $5 \%$ como sistema de retención (Fig. 1).

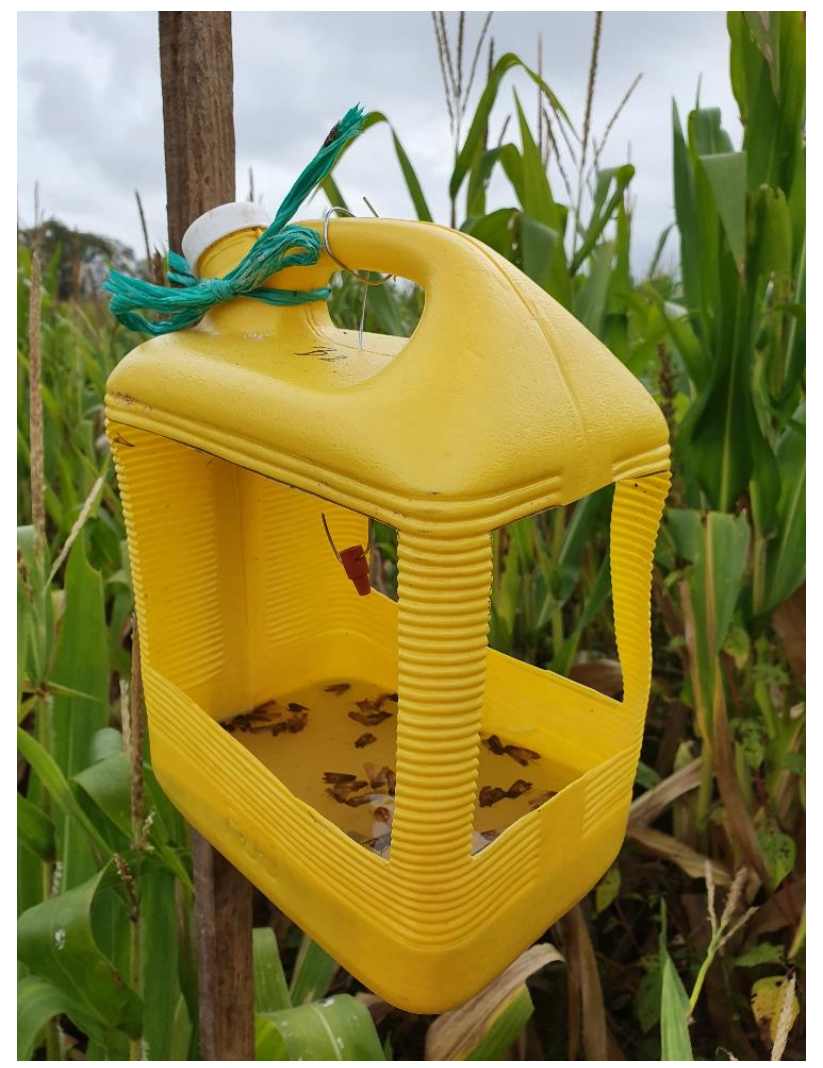

Figura 1. Trampa amarilla con el dispensador de feromona sexual instalada en campo cultivado de maíz. 
Efecto del color de trampa en las capturas. En el segundo experimento se investigó si el color de la trampa afectaba las capturas de los machos de $S$. frugiperda. En este caso, se emplearon trampas construidas con garrafas de $3.78 \mathrm{~L}$ de color amarillo, rojo, verde, azul y blanco. A cada garrafa se les hicieron tres ventanas laterales como se describe arriba y se le agregó agua jabonosa. En este experimento todas las trampas fueron cebadas con la feromona EcoTap, por ser una de las feromonas que presentó mayores capturas de machos de $S$. frugiperda en el primer experimento.

Diseño experimental. En ambos experimentos las trampas fueron colocadas en un diseño de bloques al azar con tres repeticiones, cada bloque $(100 \times 200 \mathrm{~m})$ incluyó los tratamientos correspondientes, más un control (trampa sin cebo). Estos bloques estuvieron separados por $50 \mathrm{~m}$. Las trampas fueron colocadas a $1.5 \mathrm{~m}$ sobre el suelo con ayuda de estacas de madera, con una distancia de $50 \mathrm{~m}$ entre trampa. Las trampas fueron revisadas cada tercer día y se rotaron conforme a las manecillas del reloj dentro del mismo bloque. Los insectos capturados fueron trasladados al laboratorio de Ecología del Centro Regional del Bajío del Instituto de Ecología A.C. (Pátzcuaro, Michoacán, México) en donde se identificaron, sexaron (en caso de que hubiese captura de ambos sexos) y se contabilizaron.

Análisis estadístico. Los resultados de ambos experimentos fueron analizados con el software estadístico R versión 3.6.1 (R Development Core) (Team, 2019). Las capturas obtenidas por los tratamientos evaluados fueron analizadas como número de insectos capturados/trampa/noche, usando un análisis de varianzas de medidas repetidas. El número de insectos no blanco capturados en ambos experimentos fue analizado por un modelo lineal de efectos mixtos. En el primer experimento, se dejó a los cebos feromonales evaluados y al orden de los insectos capturados como variables de efectos fijos, y a las repeticiones como efecto aleatorio. En el segundo experimento, se dejó al color de las trampas evaluadas y al orden de los insectos capturados como variables de efectos fijos, y a las repeticiones como efecto aleatorio. En ambos casos el factor respuesta fue el número de insectos capturados. Antes de los análisis se verificaron los supuestos de normalidad y homocedasticidad de los datos y, cuando fue necesario, se transformaron a través de la raíz cuadrada. Las trampas control no se tomaron en cuenta en el análisis estadístico por no presentar capturas. La comparación de medias fue realizada con la prueba de Tukey $(\alpha=0.05)$ y por comparación de intervalos de confianza.

\section{RESULTADOS}

Los experimentos fueron planeados para capturar machos de $S$. frugiperda, sin embargo, durante el trampeo de ambos experimentos se capturaron machos de Agrotis ipsilon (Hufnagel) (Lepidoptera: Noctuidae), de manera consistente, por lo que esas capturas se incluyeron en los resultados. El $70 \%$ de las palomillas capturadas correspondieron a machos de S. frugiperda, y el $30 \%$ a machos de A. ipsilon. Los insectos no blancos capturados correspondieron a tres órdenes de insectos: Diptera, Hymenoptera y Coleoptera.

Los resultados del primer experimento muestran que la captura de $S$. frugiperda fue afectada por el cebo feromonal $(\mathrm{F}=8.59 ; \mathrm{df}=4,66 ; P<0.0001)$. Las trampas cebadas con EcoTap o Phero-SF fueron las que capturaron significativamente el mayor número de machos de $S$. frugiperda, comparado con las trampas cebadas con ChemTica, Scentry o Trécé (Fig. 2A). Las capturas de machos de A. ipsilon también fueron afectadas con el cebo feromonal evaluado $(\mathrm{F}=6.71 ; \mathrm{df}=4,66 ; P=0.0023)$. Igualmente, las trampas cebadas con EcoTap o Phero-SF capturaron más machos de esta especie que las trampas cebadas con ChemTica, Scentry o Trécé, que capturaron de forma similar el menor número de machos (Fig. 2B). También encontramos que los insectos no blancos capturados por las trampas con cebos feromonales varían con respecto al tipo de orden al que pertenecen $(\mathrm{F}=10.8 ; \mathrm{df}=4,206 ; P<0.0001)$, pero no fueron afectados por el tipo de cebo feromonal ( $\mathrm{F}=1.37 ; \mathrm{df}=4,206 ; P=0.24)$. Además, la interacción entre tipo de cebo y ordenes de insectos no fue significativa $(\mathrm{F}=1.18 ; \mathrm{df}=8,206 ; P=0.31)$. El mayor número de insectos no blanco capturadas por las trampas pertenecieron al orden Diptera (Figs. 3, 4). Las trampas con EcoTap o 
Phero-SF fueron las que numéricamente presentaron mayores capturas de insectos de este orden (Fig. 3); mientras que las trampas cebadas con ChemTica, Scentry o Trécé capturaron numéricamente mayor número de coleópteros e himenópteros, en comparación con EcoTap o Phero-SF.

Los resultados del segundo experimento muestran que la captura de $S$. frugiperda no fue afectada por el color de trampa $(\mathrm{F}=2.16 ; \mathrm{df}=4,41 ; P=0.089)$. Sin embargo, numéricamente, las trampas de color amarillo y rojo capturaron más machos de $S$. frugiperda, seguida por la trampa de color verde. Las trampas de color azul y blanco son las que presentaron las menores capturas (Fig. 5A). Igualmente, el color de la trampa tampoco afectó a la captura de machos de A. ipsilon $(\mathrm{F}=0.65 ; \mathrm{df}=4,95 ; P=0.62)$. No obstante, numéricamente, las trampas de color amarillo presentaron las mayores capturas, seguidas por las trampas de color blanco o rojo (Fig. 5B). Finalmente, se encontró que la captura de insectos no blanco fue afectada por el color de las trampas $(\mathrm{F}=7.07 ; \mathrm{df}=4,131 ; P<0.001)$ (Fig. 6), y por el orden de los insectos capturados $(\mathrm{F}=14.77 ; \mathrm{df}=2,131 ; P<0.0001)$ (Fig. 7). Además, la interacción entre color de trampa y orden de insecto fue significativa $(\mathrm{F}=4.59 ; \mathrm{df}=8,131 ; P<0.0001)$. La trampa de color amarillo capturó la mayor cantidad de dípteros, seguida por la trampa de color azul, rojo y verde. La trampa de color azul capturó la mayor cantidad de himenópteros, en comparación a los otros colores evaluados (Figs. 6, 7). La trampa de color blanco fue la que capturó un menor número de insectos no objetivos de forma general, aunque capturó más coleópteros e himenópteros que la de color rojo (Fig. 6).

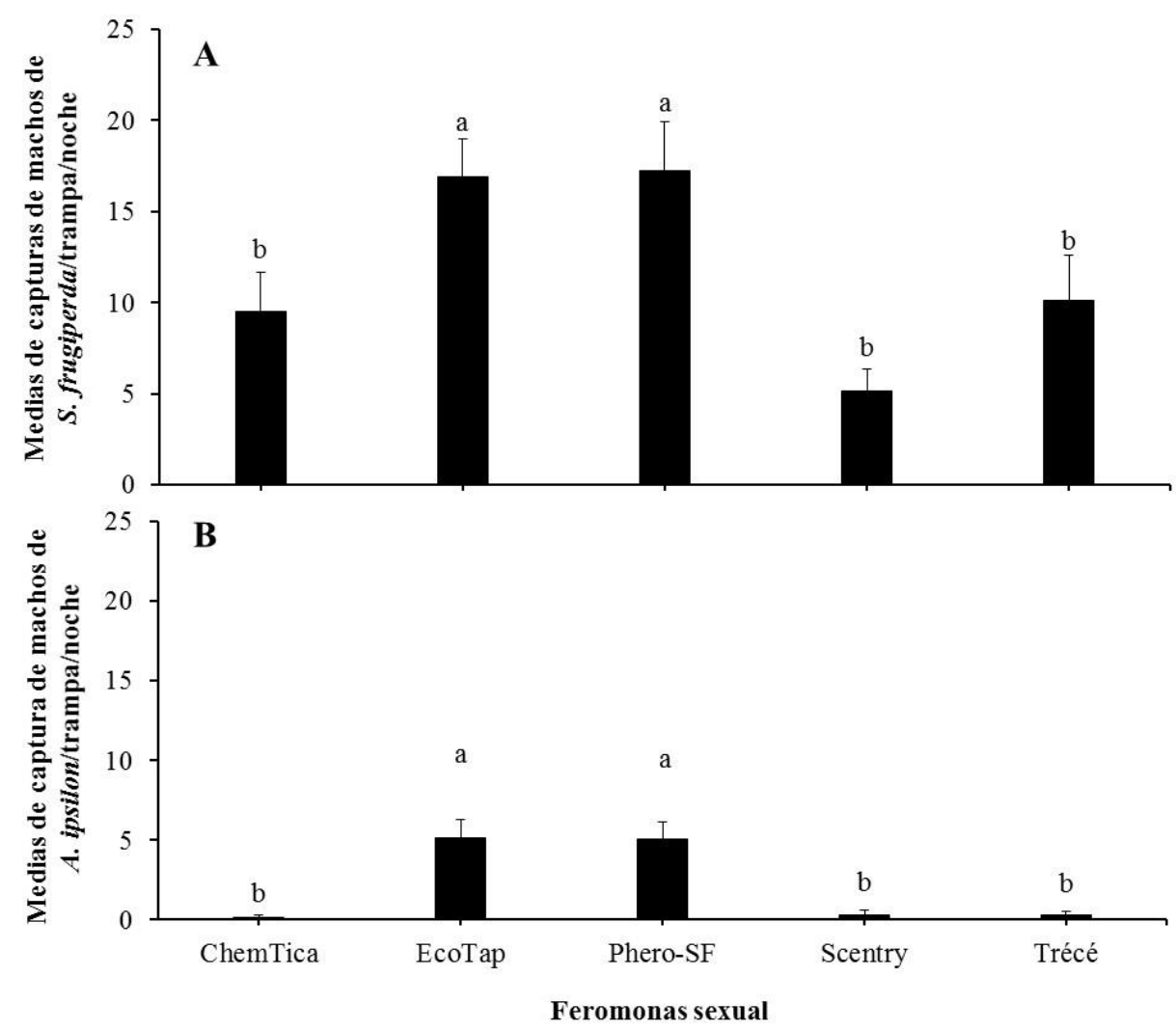

Figura 2. A) Medias \pm EE de la captura de machos de Spodoptera frugiperda por cada feromona sexual evaluada. B) Medias \pm EE de la captura de machos de Agrotis ipsilon por cada feromona sexual evaluada. Las barras con la misma letra no son significativamente diferentes (prueba de Tukey, $\alpha=0.5$ ). 


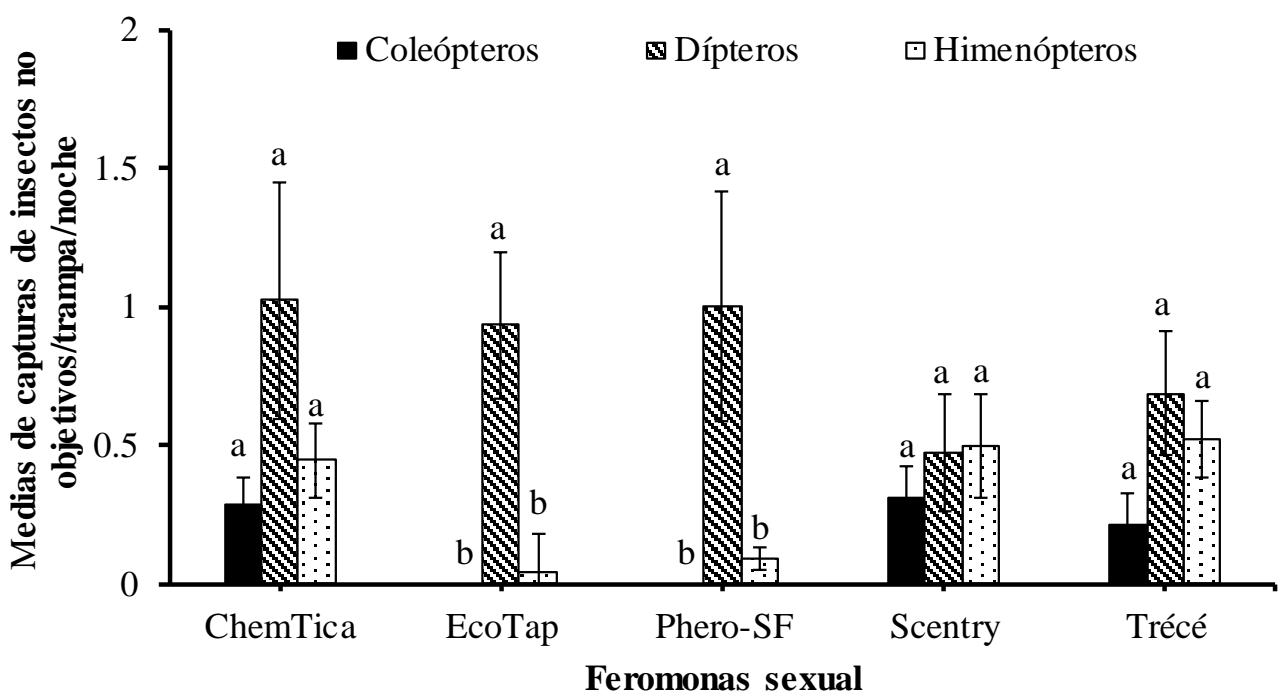

Figura 3. Medias \pm EE de la captura de machos de insectos no objetivos por cada feromona sexual evaluada comparando entre orden de insectos capturados. Las barras con la misma letra no son significativamente diferentes (prueba de Tukey, $\alpha=0.5$ ).

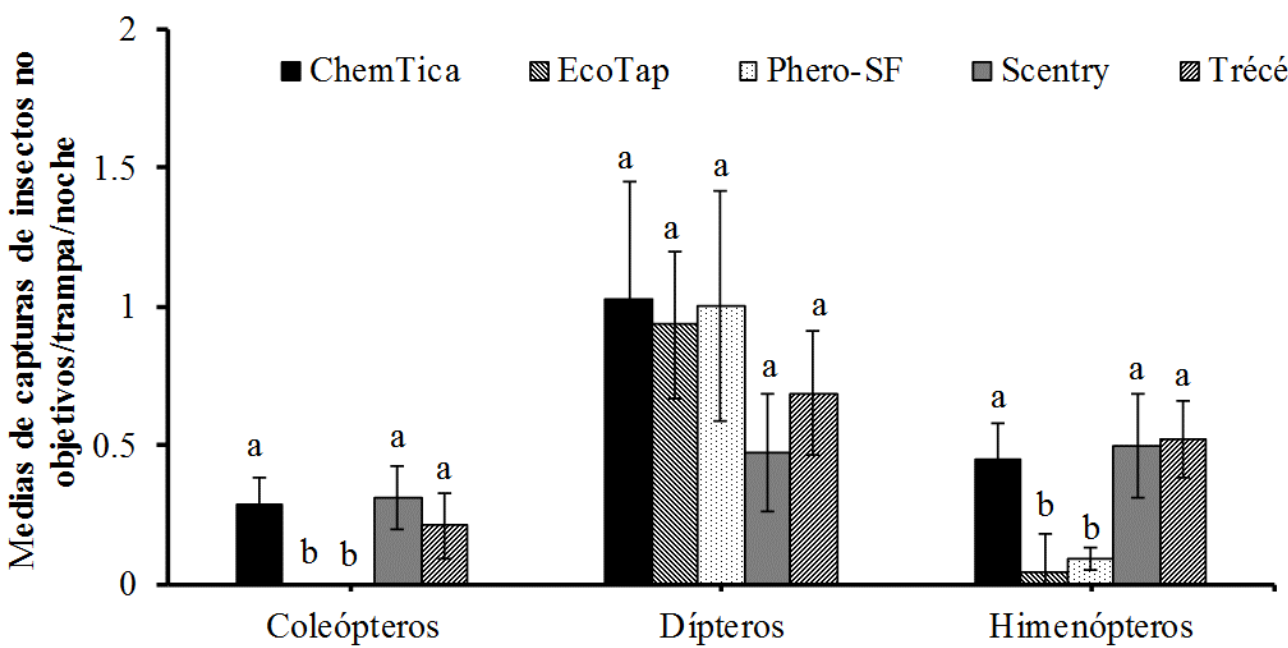

Ordenes de insectos capturados

Figura 4. Medias \pm EE de la captura de machos de insectos no objetivos por orden de insectos capturados comparando entre feromonas evaluadas. Las barras con la misma letra no son significativamente diferentes (prueba de Tukey, $\alpha=0.5$ ).

\section{DISCUSIÓN}

En este estudio encontramos que las trampas con cebo comercial Phero-SF y el cebo EcoTap fueron más efectivas en comparación con las trampas con cebos Trécé, ChemTica y Scentry para capturar machos de $S$. frugiperda. Interesantemente, estos mismos cebos fueron atractivos a machos de A. ipsilon, especie que en esta parte del país parece tener una distribución simpátrica con $S$. frugiperda. Adicionalmente, encontramos que el color de la trampa no fue un factor determinante en la captura de ambos lepidópteros, aunque si afectó la captura de los insectos no blanco. 
La variación en las capturas entre los cebos feromonales evaluados, probablemente se debe a la variación de sus componentes y a las proporciones a las que estos fueron formulados. Los cebos EcoTap y Phero-SF contienen dos acetatos, Z7-12:OAc y Z9-14:OAc en proporciones muy similares. En contraste, los cebos feromonales comerciales evaluados de ChemTica, Trécé y Scentry contienen tres acetatos, Z712:OAc, Z9-14:OAc y Z11-16:OAc en diferentes proporciones (Malo et al., 2001; Bratovich et al., 2019). Durante el llamado, las hembras de poblaciones mexicanas de $S$. frugiperda emiten los tres acetatos (Z712:OAc, Z9-14:OAc y Z11-16:OAc). Sin embargo, los machos no muestran respuestas antenales al Z1116:OAc, como lo hacen cuando son estimulados con Z7-12:OAc y Z9-14:OAc (Cruz-Esteban et al., 2018). En campos cultivados de maíz en México, solo se necesitan de esos dos compuestos (Z7-12:OAc + Z914:OAc) para capturar machos de esta plaga (Cruz-Esteban et al., datos no publicados). Estas variaciones en los componentes y en sus proporciones, podría ser la razón principal por lo que los cebos Phero-SF y EcoTap resultaron más efectivos para atraer a los machos de $S$. frugiperda en campo. Esta mezcla binaria $(Z 7-12: O A c+$ Z9-14:OAc) y en proporciones similares, también ha resultado ser más efectivas que las mezclas terciarias o cuaternarias en otras regiones como Costa Rica, Canadá, Florida, Carolina del Norte, Puerto Rico, Perú y Argentina (Andrade et al., 2000; Unbehend et al., 2014). El dispositivo que utilizan las diferentes empresas para liberar los compuestos feromonales, también puede ser un factor que afecte los resultados de captura, debido a las diferentes tasas de liberación, tal como ha sido reportado para la feromona sexual de Plutella xyloxtella (L.) (Lepidoptera: Plutellidae) (Mayer \& Mitchell, 1999). Sin embargo, en el presente estudio, esta variable no parece ser un factor importante, ya que la feromona ChemTica se libera mediante un par de membranas, mientras que las otras feromonas usan un septo de caucho, con el cual, en este estudio, se obtuvieron las mejores capturas, con la excepción de la feromona Scentry y Trécé que no difirieron estadísticamente de la feromona ChemTica.

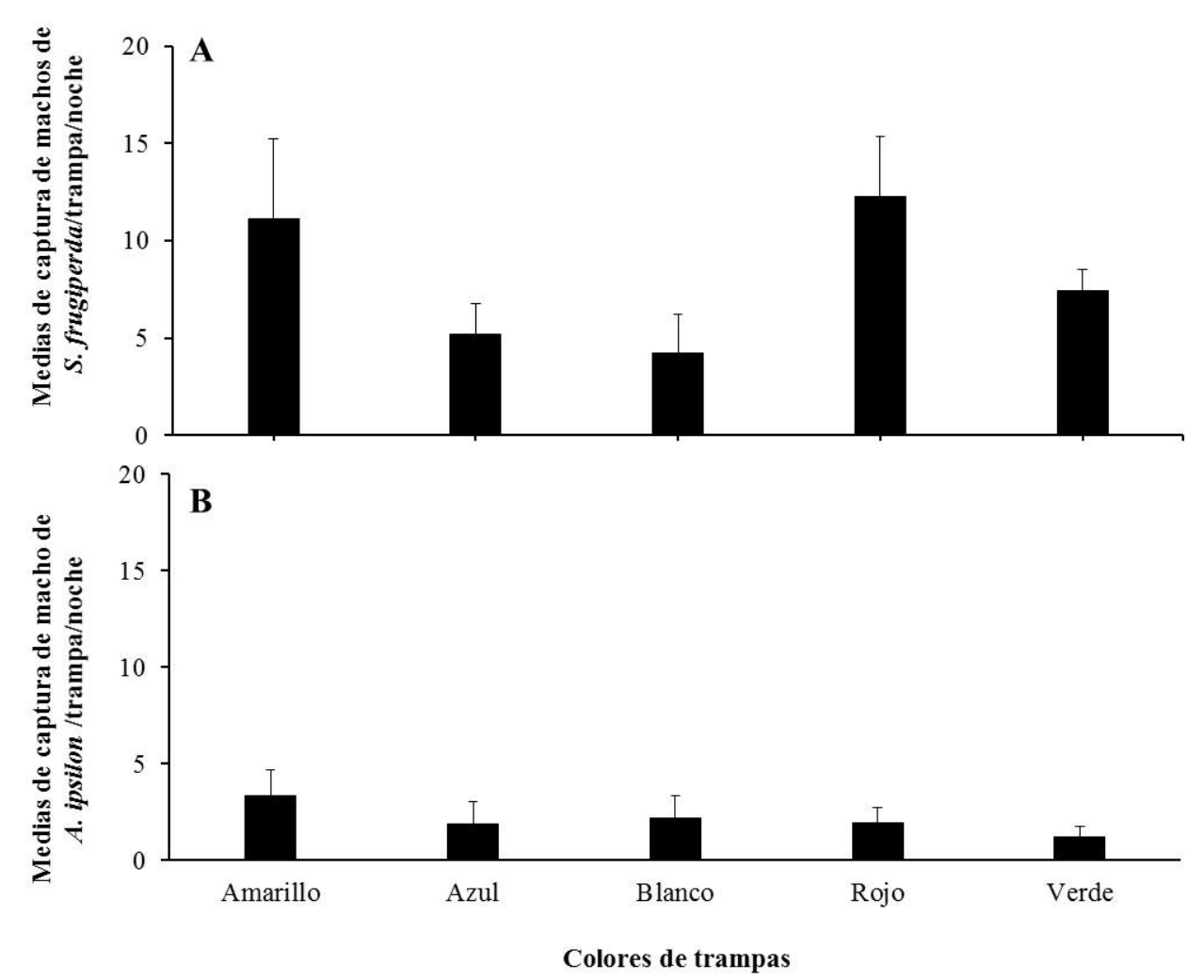

Figura 5. A) Medias \pm EE de la captura de machos de Spodoptera frugiperda por cada color de trampas evaluadas. B) Medias \pm EE de la captura de machos de Agrotis ipsilon por cada color de trampas evaluadas. Las barras con la misma letra no son significativamente diferentes (prueba de Tukey, $\alpha=0.5$ ). 
Interesantemente, los machos de A. ipsilon fueron capturados en las trampas cebadas con feromona de $S$. frugiperda. Los componentes de feromona sexual de $S$. frugiperda pueden encontrarse también en la feromona sexual de otras especies de insectos por lo que, potencialmente, puede atraer a palomillas de otras especies (Weber \& Ferro, 1991). En este caso, la feromona de A. ipsilon está constituida por los mismos tres componentes que la feromona de $S$. frugiperda, aunque las proporciones en las que están presentes en ambas especies es diferente. Por ejemplo, el acetato Z7-12:OAc es el compuesto mayoritario de la feromona de A. ipsilon, mientras que este mismo compuesto es minoritario en la feromona de $S$. frugiperda (Hill et al., 1979; Tumlinson et al., 1986). En contraste, el compuesto Z9-14:OAc es mayoritario en la mezcla feromonal de $S$. frugiperda, pero es un compuesto minoritario en la mezcla feromonal de A. ipsilon (Hill et al., 1979; Tumlinson et al., 1986). En teoría, este cambio de proporción de ambos compuestos debería impedir la atracción de machos de A. ipsilon a la feromona de $S$. frugiperda. En el presente estudio, ambos lepidópteros fueron capturados solamente por las trampas cebadas con EcoTap o Phero-SF. Sin embargo, la adición de un tercer componente podría ayudar a impedir la atracción cruzada entre especies simpátricas. Esto podría ayudar a explicar por qué las trampas con los cebos Trécé, ChemTica, o Scentry capturaron muy pocos machos de A. ipsilon. De esta forma, el acetato Z11-16:OAc está inhibiendo la atracción de machos de A. ipsilon y funcionando como una sinomona, beneficiando a ambas especies. Sin embargo, esta situación parece que no ocurre en todas las regiones en donde esta presente A. ipsilon. Por ejemplo, la adición de Z11-16:OAc a la mezcla binaria de Z7-12:OAc + Z9-14:OAc incrementa la captura de machos de A. ipsilon en Norteamérica (Wakamura et al., 1986; Gemeno \& Haynes, 1998). En contraste, la adición de Z11-16:OAc a la mezcla binaria de Z7-12:OAc + Z9-14:OAc, no muestra efecto significativo a bajas dosis, pero a dosis altas muestra un efecto inhibitorio en la captura de $S$. frugiperda (Cruz-Esteban et al., datos no publicados). Los datos del presente estudio parecen sugerir que en las regiones en donde ambas especies coexisten, la atracción de los machos de A. ipsilon es inhibida por la presencia de Z11-16:OAc, pero en donde están separadas geográficamente, la atracción de los machos no solamente no es inhibida, sino que es mejorada con la adicción de este acetato, tal como lo muestran los estudios en EUA (Gemeno \& Haynes, 1998). Sin embargo, para probar esta hipótesis, es necesario realizar estudios futuros.

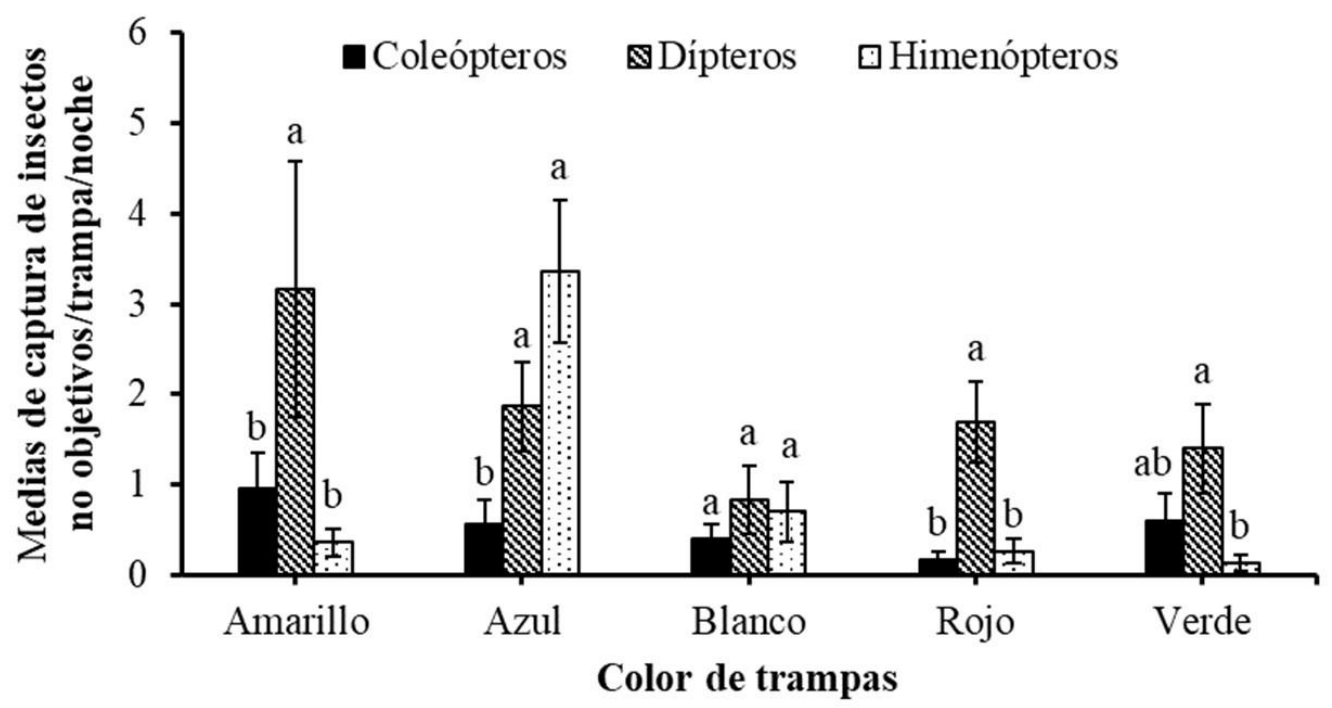

Figura 6. Medias \pm EE de la captura de machos de insectos no objetivos por cada color de trampas evaluadas comparando entre orden de insectos capturados. Las barras con la misma letra no son significativamente diferentes (prueba de Tukey, $\alpha=0.5$ ). 


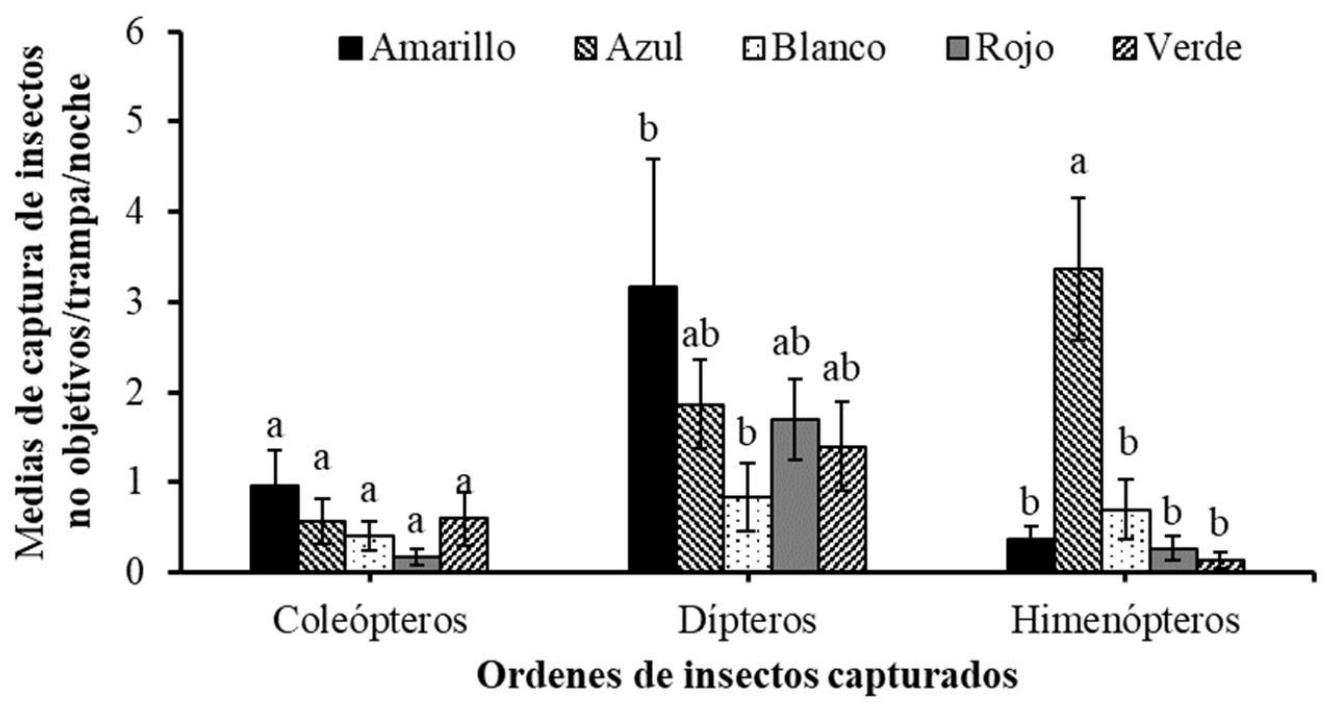

Figura 7. Medias \pm EE de la captura de machos de insectos no objetivos por orden de insectos capturados comparando entre colores de trampas evaluadas. Las barras con la misma letra no son significativamente diferentes (prueba de Tukey, $\alpha=0.5$ ).

El color, tamaño y diseño de la trampa son factores que, seguramente, están involucrados en una mayor efectividad de capturas de insectos blanco (Reardon et al., 2006; Díaz-Gómez et al., 2012; MuirheadThompson, 2012; Williams et al., 2013). Para el caso de S. frugiperda, Malo et al. (2018) reportaron que el tamaño de la trampa no es determinante para la captura de machos en campo, pero si el diseño de la trampa. Con respecto al color, estos autores reportaron que la trampa amarilla parece ser la más efectiva, y discutieron sobre un posible efecto de la densidad poblacional del insecto. También señalan que el efecto del color no quedó del todo claro, ya que el color amarillo no presentó diferencias estadísticas con respecto a los otros colores evaluados, aunque la trampa amarilla capturó un mayor número de machos de $S$. frugiperda (Malo et al., 2018). Los resultados del presente estudio son similares, ya que la trampa de color amarillo fue una de las que presentó mayor número de capturas de $S$. frugiperda, junto con la trampa de color rojo, pero sin diferencia estadística significativa. En contraste, Mitchell et al. (1989) encontraron que una trampa de dos colores (blanco y amarillo) capturó el mayor número de machos de $S$. frugiperda, en comparación con una trampa verde. Así, parece que el diseño y color de trampa están influyendo en la captura de $S$. frugiperda pero, de acuerdo con nuestros resultados, las feromonas especificas son cruciales en la captura de los machos.

En el presente estudio se encontró que las trampas control (sin cebo) capturaron muy pocos insectos no blancos, particularmente las trampas de color amarillo y azul. En estudios previos en donde han evaluado cebos feromonales de $S$. frugiperda, se ha reportado que trampas sin cebo feromonal de color blanco o amarillo, capturaron especímenes de Bombus spp. (Hamilton et al., 1971; Mitchell et al., 1989). Sin embargo, en el presente estudio se encontró un mayor número de capturas de himenópteros, dípteros y coleópteros cuando las trampas tenían feromona sexual de $S$. frugiperda, particularmente con los cebos ChemTica, Tréceé o Scentry, lo que sugiere que algún compuesto feromonal puede estar involucrado en la atracción de insectos no blanco, independientemente del color de la trampa (Gross \& Carpenter, 1991; Meagher \& Mitchell, 1999). 


\section{CONCLUSIONES}

En conclusión, encontramos que las trampas de plástico de color amarillo de $5 \mathrm{~L}$ con los cebos EcoTap o Phero-SF capturaron significativamente más machos de $S$. frugiperda y A. ipsilon que las trampas cebadas con Trécé, ChemTica, o Scentry. El color de las trampas no fue un factor determinante en la captura de ambas especies, pero con la información obtenida, es posible usar la trampa de color amarillo si deseamos obtener altas capturas de insectos plagas o la trampa de color blanco para disminuir el número de insectos benéficos atrapados, principalmente polinizadores y enemigos naturales. Este sistema de captura se puede usar para monitoreo, captura masiva tal como se ha realizado en otros lepidópteros o se puede combinar con agentes de control biológico para manejar las poblaciones del gusano cogollero en cultivos afectados, principalmente cultivos de maíz y seguir monitoreando la posible colonización en cultivos de fresas.

AgradeCimientos. Agradecemos a Christian Luis Rodríguez Enríquez, Coordinador de Investigación y Desarrollo en la empresa Koppert Development Institute, por brindarnos el área experimental. A Perla Marcelina Torres Ceja, Valentín Alonzo Servín y Sandra Sarabia Delgadillo, por el apoyo brindado en trabajo de campo y laboratorio.

\section{LITERATURA CITADA}

Aguirre, L. A., Hernández-Juárez, A., Flores, M., Cerna, E., Landeros, J., Frías, G. A., Harris, M. K. (2016) Evaluation of foliar damage by Spodoptera frugiperda (Lepidoptera: Noctuidae) to genetically modified corn (Poales: Poaceae) in Mexico. Florida Entomologist, 99 (2), 276-280. https://doi.org/10.1653/024.099.0218

Andrade, R., Rodriguez, C., Oehlschlager, A. C. (2000) Optimization of a pheromone lure for Spodoptera frugiperda (Smith) in Central America. Journal of the Brazilian Chemical Society, 11 (6), 609-613. http://dx.doi.org/10.1590/S0103-50532000000600009

Andrews, K. L. (1988) Latin American research on Spodoptera frugiperda (Lepidoptera: Noctuidae). Florida Entomologist, 71 (4), 630-653. https://www.jstor.org/stable/3495022

Baker, T. C. (2002) Mechanism for saltational shifts in pheromone communication systems. Proceedings of the National Academy of Sciences, 99 (21), 13368-13370. https://doi.org/10.1073/pnas.222539799

Batista-Pereira, L. G., Stein, K., de Paula, A. F., Moreira, J. A., Cruz, I., Figueiredo, M. L. C., Perri, Jr. J., Corrêa, A. G. (2006) Isolation, identification, synthesis, and field evaluation of the sex pheromone of the Brazilian population of Spodoptera frugiperda. Journal of Chemical Ecology, 32 (5), 1085. https://doi.org/10.1007/s10886-006-9048-5

Blanco, C. A., Pellegaud, J. G., Nava-Camberos, U., Lugo-Barrera, D., Vega-Aquino, P., Coello, J., Terán-Vargas, A. P., Vargas-Camplis, J. (2014) Maize pests in Mexico and challenges for the adoption of integrated pest management programs. Journal of Integrated Pest Management, 5 (4), E1-E9. https://doi.org/10.1603/IPM14006

Bratovich, C., Saluso, A., Murua, M. G., Guerenstein, P. G. (2019) Evaluation of sex pheromone formulations to attract Spodoptera frugiperda (Lepidoptera: Noctuidae) adult males in Argentina. Revista de la Sociedad Entomológica Argentina, 78 (3), 7-14. https://doi.org/10.25085/rsea.780302

Cardé, R. T., Minks, A. K. (1995) Control of moth pests by mating disruption: successes and constraints. Annual Review of Entomology, 40 (1), 559-585. https://doi.org/10.1146/annurev.en.40.010195.003015 
Casmuz, A., Juárez, M. L., Socías, M. G., Murúa, M. G., Prieto, S., Medina, S., Willink, E., Gastaminza, G. (2010) Revisión de los hospederos del gusano cogollero del maíz, Spodoptera frugiperda (Lepidoptera: Noctuidae). Revista de la Sociedad Entomológica Argentina, 69 (3-4), 209-231.

Clark, P. L., Molina-Ochoa, J., Martinelli, S., Skoda, S. R., Isenhour, D. J., Lee, D. J., Krumm, J. T., Foster, J. E. (2007) Population variation of the fall armyworm, Spodoptera frugiperda, in the Western Hemisphere. Journal of Insect Science, 7 (1). https://doi.org/10.1673/031.007.0501

Cruz-Esteban, S., Rojas, J. C., Sánchez-Guillén, D., Cruz-López, L., Malo, E. A. (2018) Geographic variation in pheromone component ratio and antennal responses, but not in attraction, to sex pheromones among fall armyworm populations infesting corn in Mexico. Journal of Pest Science, 91 (3), 973-983.

https://doi.org/10.1007/s10340-018-0967-z

Descoins, C., Silvain, J. F., Lalanne-Cassou, B., Cheron, H. (1988) Monitoring of crop pests by sexual trapping of males in Guadeloupe and Guyana. Agriculture, Ecosystems \& Environment, 21 (1-2), 53-56. https://doi.org/10.1016/0167-8809(88)90139-9

Díaz-Gómez, O., Malo, E. A., Patiño-Arrellano, S. A., Rojas, J. C. (2012) Pheromone trap for monitoring Copitarsia decolora (Lepidoptera: Noctuidae) activity in cruciferous crops in Mexico. Florida Entomologist, 95 (3), 602-609.

https://www.jstor.org/stable/23268483

El-Sayed, A. M., Suckling, D. M., Byers, J. A., Jang, E. B., Wearing, C. H. (2009) Potential of "lure and kill" in long-term pest management and eradication of invasive species. Journal of Economic Entomology, 102 (3), 815-835. https://doi.org/10.1603/029.102.0301

Fleischer, S. J., Harding, C. L., Blom P. E., White, J. J., Grehan, J. (2005) Spodoptera frugiperda pheromone lures to avoid nontarget captures of Leucania phragmatidicola. Journal of Economic Entomology, 98 (1), 66-71. https://doi.org/10.1093/jee/98.1.66

Ganiger, P. C., Yeshwanth, H. M., Muralimohan, K., Vinay, N., Kumar, A. R. V., Chandrashekara, K. (2018) Occurrence of the new invasive pest, fall armyworm, Spodoptera frugiperda (JE Smith) (Lepidoptera: Noctuidae), in the maize fields of Karnataka, India. Current Science, 115 (4), 621623.

Gemeno, C., Lutfallah, A. F., Haynes, K. F. (2000) Pheromone blend variation and cross-attraction among populations of the black cutworm moth (Lepidoptera: Noctuidae). Annals of the Entomological Society of America, 93 (6), 1322-1328. https://doi.org/10.1603/0013-8746(2000)093[1322:PBVACA]2.0.CO;2

Gemeno, C., Haynes, K. F. (1998) Chemical and behavioral evidence for a third pheromone component in a North American population of the black cutworm moth, Agrotis ipsilon. Journal of Chemical Ecology, 24 (6), 999-1011.

https://doi.org/10.1023/A:1022398318465

Goergen, G., Kumar, P. L., Sankung, S. B., Togola, A., Tamo, M. (2016) First report of outbreaks of the fall armyworm Spodoptera frugiperda (JE Smith) (Lepidoptera, Noctuidae), a new alien invasive pest in West and Central Africa. PloS One, 11 (10), e0165632. https://doi.org/10.1371/journal.pone.0165632

Groot, A. T., Inglis, O., Bowdridge, S., Santangelo, R. G., Blanco, C., López, Jr, J. D., Terán Vargas, A., Gould, F., Schal, C. (2009) Geographic and temporal variation in moth chemical communication. Evolution: International Journal of Organic Evolution, 63 (8), 1987-2003. https://doi.org/10.1111/j.1558-5646.2009.00702.x

Gross, H. R., Carpenter, J. E. (1991) Role of the fall armyworm (Lepidoptera: Noctuidae) pheromone and other factors in the capture of bumblebees (Hymenoptera: Aphidae) by universal moth traps. 
Environmental Entomology, 20 (1), 377-381.

https://doi.org/10.1093/ee/20.1.377

Hamilton, D. W., Schwartz, P. H., Townshend, B. G., Jester, C. W. (1971) Effect of color and design of traps on captures of Japanese beetles and bumblebees. Journal of Economic Entomology, 64 (2), 430-432. https://doi.org/10.1093/jee/64.2.430

Hardke, J. T., Leonard, B. R., Huang, F., Jackson, R. E. (2011) Damage and survivorship of fall armyworm (Lepidoptera: Noctuidae) on transgenic field corn expressing Bacillus thuringiensis Cry proteins. Crop Protection, 30 (2), 168-172. https://doi.org/10.1016/j.cropro.2010.10.005

Hill, A. S., Rings, R. W., Swier, S. R., Roelofs, W. L. (1979) Sex pheromone of the black cutworm moth, Agrotis ipsilon. Journal of Chemical Ecology, 5 (3), 439-457. https://doi.org/10.1007/BF00987929

Kamarudin, N., Ahmad, S. N., Arshad, O., Wahid, M. B. (2010) Pheromone mass trapping of bagworm moths, Metisa plana Walker (Lepidoptera: Psychidae), for its control in mature oil palms in Perak, Malaysia. Journal of Asia-Pacific Entomology, 13 (2), 101-106. https://doi.org/10.1016/j.aspen.2009.11.003

Larraín, S. P., Guillon, M., Kalazich, J., Graña, F., Vásquez, C. (2009) Effect of pheromone trap density on mass trapping of male potato tuber moth Phthorimaea operculella (Zeller) (Lepidoptera: Gelechiidae), and level of damage on potato tubers. Chilean Journal of Agricultural Research, 69 (2), 281-285.

Mafra-Neto, A., Habib, M. (1996) Evidence that mass trapping suppresses pink bollworm populations in cotton fields. Entomologia Experimentalis et Applicata, 81 (3), 315-323. https://doi.org/10.1046/j.1570-7458.1996.00102.x

Malo, E. A., Cruz-Esteban, S., González, F. J., Rojas, J. C. (2018) A home-made trap baited with sex pheromone for monitoring Spodoptera frugiperda males (Lepidoptera: Noctuidae) in corn crops in Mexico. Journal of Economic Entomology, 111 (4), 1674-1681. https://doi.org/10.1093/jee/toy 128

Malo, E. A., Cruz-Lopez, L., Valle-Mora, J., Virgen, A., Sanchez, J. A., Rojas, J. C. (2001) Evaluation of commercial pheromone lures and traps for monitoring male fall armyworm (Lepidoptera: Noctuidae) in the coastal region of Chiapas, Mexico. Florida Entomologist, 84 (4), 659-664.

Matsuoka, Y., Vigouroux, Y., Goodman, M. M., Sanchez, J., Buckler, E., Doebley, J. (2002) A single domestication for maize shown by multilocus microsatellite genotyping. Proceedings of the National Academy of Sciences, 99 (9), 6080-6084. https://doi.org/10.1073/pnas.052125199

Mayer, M. S., Mitchell, E. R. (1999) Differences between attractive diamondback moth, Plutella xylostella (L.) (Lepidoptera: Plutellidae), sex pheromone lures are not determinable through analysis of emissions. Agricultural and Forest Entomology, 1 (3), 229-236. https://doi.org/10.1046/j.1461-9563.1999.00031.x

Meagher Jr, R. L., Mitchell, E. R. (1999) Nontarget Hymenoptera collected in pheromone-and synthetic floral volatile-baited traps. Environmental Entomology, 28 (3), 367-371. https://doi.org/10.1093/ee/28.3.367

Mitchell, E. R., Agee, H. R., Heath, R. R. (1989) Influence of pheromone trap color and design on capture of male velvetbean caterpillar and fall armyworm moths (Lepidoptera: Noctuidae). Journal of Chemical Ecology, 15 (6), 1775-1784. https://doi.org/10.1007/BF01012265

Montezano, D. G., Specht, A., Sosa-Gómez, D. R., Roque-Specht, V. F., de Paula-Moraes, S. V., Peterson, J. A., Hunt, T. E. (2019) Developmental parameters of Spodoptera frugiperda (Lepidoptera: Noctuidae) immature stages under controlled and standardized conditions. Journal of Agricultural Science (Toronto), 11 (8), 76-89. https://doi.org/10.5539/jas.v11n8p76 
Muirhead-Thompson, R. C. (2012) Trap responses of flying insects: the influence of trap design on capture efficiency. Academic Press, London, San Diego, New York, 304 pp.

Oerke, E. C. (2006) Crop losses to pests. The Journal of Agricultural Science, 144 (1), 31-43. https://doi.org/10.1017/S0021859605005708

Pashley, D. P. (1986) Host-associated genetic differentiation in fall armyworm (Lepidoptera: Noctuidae): a sibling species complex? Annals of the Entomological Society of America, 79 (6), 898-904. https://doi.org/10.1093/aesa/79.6.898

Phelan, P. L. (1992) Evolution of sex pheromones and the role of asymmetric tracking, Pp: 265-314. En: B. D. Roitberg, M. B. Isman (Eds.) Insect chemical ecology: an evolutionary approach. Chapman \& Hall, New York, London.

Prowell, D. P., McMichael, M., Silvain, J. F. (2004) Multilocus genetic analysis of host use, introgression, and speciation in host strains of fall armyworm (Lepidoptera: Noctuidae). Annals of the Entomological Society of America, 97 (5), 1034-1044. https://doi.org/10.1603/0013-8746(2004)097[1034:MGAOHU]2.0.CO;2

Reardon, B. J., Sumerford, D. V., Sappington, T. W. (2006) Impact of trap design, windbreaks, and weather on captures of European corn borer (Lepidoptera: Crambidae) in pheromone-baited traps. Journal of Economic Entomology, 99 (6), 2002-2009. https://doi.org/10.1093/jee/99.6.2002

SENASICA (2019) Berries mexicanas, caso de éxito. Servicio Nacional de Sanidad, inocuidad y Calidad Agroalimentaria. Disponible en: https://www.gob.mx/senasica/articulos/berries-mexicanas-casode-exito (última consulta septiembre 2019).

Shylesha, A. N., Jalali, S. K., Gupta, A., Varshney, R., Venkatesan, T., Shetty, P., Ojha, R., Ganiger, P. C., Navik, O., Subaharan, K., Bakthavatsalam, N., Ballal, C. R., Raghavendra, A. (2018) Studies on new invasive pest Spodoptera frugiperda (JE Smith) (Lepidoptera: Noctuidae) and its natural enemies. Journal of Biological Control, 32 (3), 1-7. https://doi.org/10.18311/jbc/2018/21707

Smadja, C., Butlin, R. K. (2009) On the scent of speciation: the chemosensory system and its role in premating isolation. Heredity, 102 (1), 77-97. https://doi.org/10.1038/hdy.2008.55

Solano, Y., Sosa, F., de Camacaro, M. P. (2015) Registros de noctuidos (Lepidoptera: Noctuidae) asociados al cultivo de fresa en el occidente de Venezuela. Entomotropica, 30 (19), 193-200.

Stokstad, E. (2017) New crop pest takes Africa at lightning speed. Science, 356 (6337), 473-474. https://doi.org/10.1126/science.356.6337.473

Swamy, H. M. M., Asokan, R., Kalleshwaraswamy, C. M., Sharanabasappa, K., Prasad, Y. G., Maruthi, M. S., Shashank, P., Devi, N. I., Surakasula, A., Reddy, G. S. S., Nagesh, S. N. (2018) Prevalence of "R" strain and molecular diversity of fall armyworm Spodoptera frugiperda (J. E. Smith) (Lepidoptera: Noctuidae) in India. Indian Journal Entomolgy, 80 (3), 544-553. https://doi.org/10.5958/0974-8172.2018.00239.0

Tamez Guerra, P., Galán Wong, L. J., Medrano Roldán, H., García Gutiérrez, C., Rodríguez Padilla, C., Gómez Flores, R. A., Tamez Guerra, R. S. (2001) Bioinsecticidas: su empleo, producción y comercialización en México. Ciencia UANL, 4 (2), 143-152.

Team, R. C. (2019) R: A language and environment for statistical computing. R Foundation for Statistical Computing, Vienna, Austria. Disponible en: http://www.R-proje ct.org/

Tòth, M., Löfstedt, C., Blair, B. W., Cabello, T., Farag, A. I., Hansson, B. S., Kovalec, B. G., Maini, S., Nesterov, E. A., Pajor, I., Sazonov, A. P., Shamshev, I. V., Subchev, M., Szöcs, G. (1992) Attraction of male turnip moths Agrotis segetum (Lepidoptera: Noctuidae) to sex pheromone components and their mixtures at 11 sites in Europe, Asia, and Africa. Journal of Chemical Ecology, 18 (8), 1337-1347. https://doi.org/10.1007/BF00994360

Tumlinson, J. H., Mitchell, E. R., Teal, P. E. A., Heath, R. R., Mengelkoch, L. J. (1986) Sex pheromone of fall armyworm, Spodoptera frugiperda (JE Smith). Journal of Chemical Ecology, 12 (9), 1909- 
1926.

https://doi.org/10.1007/BF01041855

Unbehend, M., Hänniger, S., Vásquez, G. M., Juárez, M. L., Reisig, D., McNeil, J. N., Meager, R. L., Jenkins, D. A., Heckel, D. G., Groot, A. T. (2014) Geographic variation in sexual attraction of Spodoptera frugiperda corn-and rice-strain males to pheromone lures. PloS One, 9 (2), e89255. https://doi.org/10.1371/journal.pone.0089255

Wakamura, S., Struble, D. L., Matsuura, H., Sato, M., Kegasawa, K. (1986) Sex pheromone of the black cutworm moth, Agrotis ipsilon Hufnagel (Lepidoptera: Noctuidae): Attractant synergist and improved formulation. Applied Entomology and Zoology, 21 (2), 299-304. https://doi.org/10.1303/aez.21.299

Weber, D. C., Ferro, D. N. (1991) Nontarget noctuids complicate integrated pest management monitoring of sweet corn with pheromone traps in Massachusetts. Journal of Economic Entomology, 84 (4), $1364-1369$. https://doi.org/10.1093/jee/84.4.1364

Williams, D. T., Straw, N., Townsend, M., Wilkinson, A. S., Mullins, A. (2013) Monitoring oak processionary moth Thaumetopoea processionea L. using pheromone traps: the influence of pheromone lure source, trap design and height above the ground on capture rates. Agricultural and Forest Entomology, 15 (2), 126-134. https://doi.org/10.1111/afe.12005

Witzgall, P., Kirsch, P., Cork, A. (2010) Sex pheromones and their impact on pest management. Journal of Chemical Ecology, 36 (1), 80-100. https://doi.org/10.1007/s10886-009-9737-y 